\title{
PEDAGOGIA HOSPITALAR: CONCEITO E IMPORTÂNCIA FRENTE AOS DIREITOS DA CRIANÇA HOSPITALIZADA
}

\author{
Letícia Meireles de Souza ${ }^{1}$ \\ Gleicieli Karine dos Reis Dias ${ }^{2}$ \\ Fabrícia Lino da Silva ${ }^{3}$ \\ Claudia Lopes Perpétuo ${ }^{4}$
}

SOUZA, L. M. de; DIAS, G. K. dos R.; SILVA, F. L. da; PERPÉTUO, C. L. Pedagogia hospitalar: conceito e importância, frente aos direitos da criança hospitalizada. EDUCERE - Revista da Educação, Umuarama, v. 18, n. 1, p. 81-92, jan./jun. 2018.

RESUMO: O presente artigo possui o propósito de conceituar a pedagogia hospitalar, compreender sua origem e destacar o papel fundamental do pedagogo neste ambiente. Sendo assim, este profissional auxilia no desenvolvimento das capacidades dos indivíduos que se encontram nos hospitais, ou seja, possuem grande importância no processo ensino-aprendizagem. É, salientado, aspectos frente aos direitos das crianças hospitalizadas, considerando seus cuidados e questões pertinentes. Para tanto serão abordados resultados de uma pesquisa bibliográfica acerca da pedagogia hospitalar, um assunto de considerável valor que precisa ser mais estudado.

PALAVRAS-CHAVE: Educação; Pedagogia hospitalar; Pedagogo.

\section{HOSPITAL PEDAGOGY: CONCEPT AND IMPORTANCE IN FACE OF THE RIGHTS OF THE HOSPITALIZED CHILD}

\footnotetext{
ABSTRACT: This article has the purpose of conceptualizing hospital

DOI: $10.25110 /$ educere.v18i1.2018.6797

${ }^{1}$ Acadêmica do Curso de Pedagogia da UNIPAR - Universidade Paranaense. Avenida Londrina, 3.340. CEP: 87502-250. Umuarama - Pr. leticialms09@gmail.com

${ }^{2}$ Acadêmica do Curso de Pedagogia da UNIPAR - Universidade Paranaense. Rua Santa Amélia, 463. CEP: 87400-000. Cruzeiro do Oeste - Pr. gleycy_cdo@hotmail.com

${ }^{3}$ Acadêmica do Curso de Pedagogia da UNIPAR - Universidade Paranaense. Rua Jacarandá, 339. CEP: 87538-000. Perobal - Pr. fabricia_lino16@hotmail.com

${ }^{4}$ Mestre em Psicologia pela Pontifícia Universidade Católica do Rio Grande do Sul (2003). Atualmente é professora titular - regime Tempo Integral da Universidade Paranaense. E-mail: clauperpetuo@prof.unipar.br
} 
pedagogy, understanding its origin and highlighting the key role of the pedagogue in such environment. Therefore, this professional helps in developing the capacities of individuals who are hospitalized, that is, they have a great importance in the teaching-learning process. Therefore, the results of a literature review will be discussed regarding hospital pedagogy, a considerable valuable matter that needs to be further studied.

KEYWORDS: Education; Hospital pedagogy; Pedagogue.

\section{PEDAGOGÍA HOSPITALARIA: CONCEPTO E IMPORTANCIA, FRENTE A LOS DERECHOS DEL NIÑO HOSPITALIZADO}

RESUMEN: El presente artículo tiene el propósito de conceptualizar la pedagogía hospitalaria, comprender su origen y destacar el papel fundamental del pedagogo en este ambiente. Por lo tanto, este profesional ayuda en el desarrollo de las capacidades de los individuos que se encuentran en los hospitales, o sea, tienen gran importancia en el proceso enseñanza y aprendizaje. Se destaca, aspectos frente a los derechos de los niños hospitalizados, considerando sus cuidados y cuestiones pertinentes. Para ello se abordarán resultados de una investigación bibliográfica acerca de la pedagogía hospitalaria, un asunto de considerable valor que necesita ser más estudiado.

PALABRAS CLAVE: Educación; Pedagogía hospitalaria; Pedagogo.

\section{INTRODUÇÃO}

A educação pode ocorrer em diversos lugares, desde situações fortuitas, até em locais especializados, como é o caso das instituições de ensino, porém quando pensamos em crianças hospitalizadas surge certa indagação sobre como aprendem os conhecimentos formais transmitidos no âmbito escolar.

Neste sentido, este artigo visa explanar direitos da criança hospitalizada, bem como, considerar aspectos de uma das áreas de atuação do pedagogo com o intuito de ampliar os conhecimentos necessários para essa atuação, destacar o papel do pedagogo, a origem da pedagogia hospitalar e seu conceito.

Sendo assim é preciso compreender que a pedagogia hospitalar é um termo que remete ao atendimento educacional dos educandos que 
encontram-se em situação hospitalar, tendo por objetivo promover e aprimorar as capacidades cognitivas deste, porém é fundamental considerar o ser de maneira ampla para melhor desenvolver as funções que o pedagogo pretende alcançar.

De acordo com Esteves(2008) este ramo da pedagogia surgiu na cidade de Paris da França por volta de 1935, onde deparou-se com a demanda de promover o atendimento educacional das crianças e adolescentes com doenças que as impediam de frequentar a escola, e se popularizou no fim da Segunda Guerra Mundial, com o fato de um grande número de educandos terem sido atingidos pelo conflito, logo estes necessitavam de atendimento especializado.

Para tanto, surge questões voltados aos direitos de tais sujeitos, em que propõem um pensar agregando a necessidade de refletir, conhecer sobre os cuidados pertinentes, frente a necessidade do mesmo, em relação à hospitalização e qual respaldo legal este possui.

Dessa maneira a pedagogia hospitalar vem ao encontro com estes sujeitos que estão hospitalizados. A pedagogia hospitalar é fundamental para estes alunos, pois irá auxiliá-los na elaboração de seu próprio saber, em que o pedagogo atua como mediador do aprendizado dos conteúdos atrelados à realidade atual do educando. Freitas(2005) destaca o papel da educação e do professor neste processo:

O papel da educação no hospital e, com ela, o do professor, é propiciar a criança o conhecimento e a compreensão daquele espaço, ressignificando não somente a ele, como a própria criança, sua doença e suas relações nessa nova situação de vida. A escuta pedagógica surge, assim, como uma metodologia educativa própria do que chamamos pedagogia hospitalar. Seu objetivo é acolher a ansiedade e as dúvidas da criança hospitalizada, criar situações coletivas de reflexão sobre elas, construindo novos conhecimentos que contribuam para uma nova compreensão de sua existência, possibilitando a melhora do seu quadro clínico. (FREITAS 2005, p. 135 apud CASTRO 2009, p. 47).

Nesta perspectiva, é necessário ter um olhar diferente para esta área que precisa conciliar a educação com a condição que o sujeito se encontra, ou seja, o professor precisa compreender as especificidades dos 
educando, para que o trabalho neste local seja efetivo e concreto. Dessa forma, o professor pode trabalhar de maneira diversificada para que os conhecimentos sejam significativos.

Para tal conhecimento e a partir de uma pesquisa bibliográfica, buscou-se primeiramente a origem e o conceito da pedagogia hospitalar, objetivando investigar mais a função do papel do pedagogo nesta área de grande interesse e importância para esse profissional.

\section{Origem e conceito}

De acordo com Matos e Mugiatti, entende-se por pedagogia hospitalar:

[...]aquele ramo da Pedagogia, cujo objeto de estudo, investigação e dedicação é a situação do estudante hospitalizado, a fim de que continue progredindo na aprendizagem cultural, formativa e muito especialmente, quanto ao modo de enfrentar a sua enfermidade, com vistas ao autocuidado e à prevenção de outras possíveis alterações na sua saúde. MATOS e MUGIATTI (2009, p.79)

Neste sentido, a pedagogia hospitalar vem propor maneiras diversificadas de trabalhar com o sujeito que encontra-se em hospitais para melhor desenvolver habilidades que estes possuem.

A Pedagogia hospitalar teve início em 1935 na França, quando Henri Sallier inaugurou a primeira escola para crianças inadaptadas. Com o impacto de sua iniciativa esta prática se espalhou pela França e em outros países da Europa como Alemanha, mais tarde chegando ao continente americano, inicialmente nos Estados Unidos, "com o objetivo de suprir as dificuldades escolares de crianças tuberculosas." (ESTEVES,2008, p.02).

A Segunda Guerra Mundial também foi fundamental na criação dessas escolas em hospitais, pois de acordo com Esteves(2008) havia um grande número de crianças e adolescentes que foram atingidos, sendo impossibilitados de frequentar as escolas regulares.

No Brasil de acordo com Lima e Paleologo(2012) surgiu o movimento da Pedagogia Hospitalar na década de 50 na cidade do Rio de Janeiro na qual permanece atualmente com o atendimento aos educandos 
hospitalizados.

Segundo Matos e Mugiatti(2007), existe no estado do Paraná, um projeto direcionado na área da educação e saúde, nomeado "Hospitalização Escolarizada", em que esse projeto influenciou na implantação da Pedagogia Hospitalar no Brasil, com suporte das Secretarias da Educação e Saúde.

Nesta perspectiva, o Ministério da Educação vê a necessidade de produzir um documento nesta área com o auxílio da Secretaria Especial, que contém estratégias e orientações para proporcionar a promoção do atendimento pedagógico em classes hospitalares.

O Brasil reconheceu a legislação por meio do Estatuto da Criança e do Adolescente Hospitalizado, a Resolução n ${ }^{\circ} .41$ de outubro e 1995, no item 9, o "Direito de desfrutar de alguma forma de recreação, programas de educação para a saúde, acompanhamento do currículo escolar durante sua permanência hospitalar. DIÁRIO OFICIAL DA UNIÃO, 17/10/95 Seção I, p.163/9-16320 - Brasília - Distrito Federal. Portanto, a legislação assegura aos educandos em situação hospitalar que sejam atendidos de acordo com suas especificidades.

A Pedagogia Hospitalar, tem a responsabilidade e a obrigação de garantir apoio educacional não somente às crianças que têm transtornos do desenvolvimento, mas também às crianças e aos adolescentes que se encontram em situações de riscos ao desenvolvimento físico, psíquico, imunológico, e que se encontram afastados do convívio social e escolar.

Em 2002, é promulgado outro documento intitulado "Classe Hospitalar e Atendimento Pedagógico Domiciliar: estratégias e orientações”, publicado pelo MEC (BRASIL, 2002), com o propósito específico de estruturar ações, políticas de organização do sistema de atendimento educacional em ambientes hospitalares e domiciliares.

Cumpre às classes hospitalares e ao atendimento pedagógico domiciliar elaborar estratégias e orientações para possibilitar o acompanhamento pedagógico-educacional do processo de desenvolvimento e construção do conhecimento de crianças, jovens e adultos matriculados ou não nos sistemas de ensino regular, no âmbito da educação básica e que encontram-se impossibilitados de frequentar escola, temporária ou permanentemente e, garantir a manutenção do vínculo com as escolas por 
meio de um currículo flexibilizado e/ou adaptado, favorecendo seu ingresso, retorno ou adequada integração ao seu grupo escolar correspondente, como parte do direito de atenção integral. (BRASIL, 2002, p. 13)

Então, o objetivo da Pedagogia Hospitalar é defender o direito de toda criança e adolescente, lutando pelo respeito e cidadania, onde todos devem ter oportunidades iguais, buscando superar as dificuldades e possibilitar um ambiente propício para o aprendizado, em que busca integrar o doente em sua situação hospitalar.

Para entender melhor a pedagogia hospitalar passamos a falar agora do papel do pedagogo, visto ser este o nosso objetivo, entender qual é a função e a importância do pedagogo na pedagogia hospitalar.

\section{Direitos da criança hospitalizada.}

Leiden, 1988, em “A Carta da Criança Hospitalizada”, vincula os direitos, em que propõem cuidados a serem desenvolvidos para com a criança hospitalizada. Neste sentido, foram desenvolvidas reflexões acerca de várias associações europeias, na qual foram organizadas neste local (Leiden). Assim, estabelece alguns princípios, como: A criança deve estar em um ambiente hospitalar apenas quando os cuidados não poderão ser efetivados em casa. É direito da criança, que estejam próximas de seus pais ou de seus responsáveis, para que sintam-se melhores durante o tratamento. É direito, que tanto as crianças, quanto dos pais recebam informações sobre o estado de saúde, bem como quais os tratamentos adequados.

Para tanto, cabe destacar que em 1959, foi proclamada, a Declaração dos Direitos da Criança, durante a 841. a sessão plenária da Assembleia Geral das Nações Unidas, tal documento possui 10 princípios que abarcam os direitos pertinentes à criança, voltado ao que deve-se aplicar para que tenham uma infância mais segura, para considerar suas necessidades. No Princípio $4^{\circ}$ afirma que:

A criança deve beneficiar da segurança social. Tem direito a crescer e a desenvolver-se com boa saúde; contribuindo para este fim, deverão ser proporcionados, quer à criança, quer à sua mãe, cuidados especiais, designadamente, tratamento pré 
e pós-natal. A criança tem direito a uma adequada alimentação, habitação, recreio e cuidados médicos.

Outro aspecto de grande importância, seria o Conselho Nacional dos Direitos da Criança e Adolescente, em que propõem assegurar às crianças hospitalizadas o direito e proteção a sua vida e saúde, bem como todo apoio necessário para que se sintam seguras e em condições agradáveis durante sua estadia no hospital. Destaca-se então que as crianças tem: "Direito a desfrutar de alguma forma de recreação, programas de educação para a saúde, acompanhamento do currículo escolar, durante sua permanência hospitalar”. CONANDA (BRASIL, 1995).

Para tanto, como salienta Levy, M. L. (1996): “Os direitos da criança hospitalizada são apenas uma pequena parte dos direitos que, ao longo dos anos, têm sido concedidos à Criança". Dessa maneira, é possível perceber que é necessário agregar e ampliar tais questões, buscando que os direitos sejam considerados e efetivados.

\section{O papel do pedagogo}

A Pedagogia Hospitalar é uma prática que visa assessorar crianças e jovens que não estão em condições de frequentar a escola. O professor/pedagogo possui papel de extrema importância, pois vai auxiliar o aluno para que dê continuidade aos estudos e assim não seja prejudicado devido à condição que se encontra.

Essas crianças/jovens vivem um momento difícil de sua vida e essa situação tende a alterar seu desenvolvimento, é nesse âmbito que o pedagogo assume um papel de agente transformador, de levar a esses pacientes atividades que proporcionem a continuação de sua aprendizagem com conteúdos escolares e lúdicos. "A pedagogia hospitalar aponta, ainda mais um recurso contributivo para a cura. Favorece a associação do resgate, de forma multidisciplinar, da condição inata do organismo, de saúde e bem-estar, ao resgate da humanização e da cidadania." (Matos, 2006, p. 29)

Neste sentido, há várias leis que enfatizam o direito a escolarização em ambientes hospitalares, como a Lei 1. 044/ 69, diz que toda criança e adolescente hospitalizado tenha direito a educação, assim como a Lei Federal $n^{0} 11$. 104, de 21/03/2005, que obriga as enfermarias pediátricas a 
implantarem brinquedotecas e classes hospitalares, que é um dos serviços da Modalidade da Educação Especial.

Segundo Fonseca(1999) ainda não se pode afirmar se esses serviços realmente já se concretizaram nos hospitais, mas vale ressaltar que mesmo com todas essas leis a favor da educação em hospitais, sua efetivação dependerá da disponibilidade dos hospitais, ou seja, se haverá espaço físico e o tipo de convênio.

É ideal que o professor hospitalar ao planejar as atividades que serão ministradas leve em consideração não só o ambiente (seja no leito ou em classe hospitalar) como também o contexto do aluno, tendo em conta o nível de aprendizagem e faixa etária, na qual geralmente se diversifica nesses ambientes.

A classe hospitalar contemporânea, além de atender às necessidades pedagógicas- educacionais da criança e do adolescente hospitalizado (necessidades provenientes da atenção integral ao seu desenvolvimento), obedece aos fundamentos políticos da educação, isto é, ratifica o respeito aos princípios democráticos da igualdade, da liberdade e da valorização da dignidade humana. (Fonseca, 1999, p. 31)

Torna- se fundamental que o pedagogo esteja devidamente habilitado a trabalhar com a diversidade e diferentes experiências, para que assim possa identificar as necessidades educacionais de cada paciente. Pimenta (1997) destaca que o professor pode utilizar da ludicidade, possuindo roupas diferenciadas para não assemelhar-se com o médico. Sua linguagem deve ser de maneira informal ao desempenhar seu trabalho.

Para Matos e Mugiatti(2001) vale ressaltar o valor da cooperação de equipes médicas, psicólogos e assistentes sociais para com o professor, afim de assegurar uma conciliação das situações problematizadas, alcançando níveis de melhorias tanto na saúde, quanto na aprendizagem.

Há vários métodos que o pedagogo pode utilizar para propiciar a aprendizagem nesses pacientes, dentre elas o lúdico é o mais abordado, por meio das brincadeiras, jogos, para que os educandos exercitem sua criatividade e também sintam prazer em estudar. Neste sentido, Froebel(1912) destaca a importância do brincar para melhor desenvolver os educandos: "A brincadeira é a atividade espiritual mais pura do homem 
[...] Ela dá alegria, liberdade, contentamento, descanso externo e interno, paz com o mundo... O Brincar em qualquer tempo não é trivial, é altamente sério e de profunda significação.” (Froebel, 1912)

À vista disso, é possível perceber que o brincar é importante para o desenvolvimento das crianças, pois com esta prática elas irão desenvolver suas capacidades e possuir uma melhor qualidade de vida, como diz Frebel(1912) que o brincar é necessário e sério e que possibilita o aprimoramento da aprendizagem da criança.

Então, o pedagogo deve repensar sua didática com ações pedagógicas, em que tenha um começo e fim no mesmo dia, já que em determinados casos, os pacientes podem ter alta, ou devido ao tratamento e cirurgias, os quais poderiam deixar os pacientes sem condições de se descolocar às classes.

O pedagogo deve oferecer uma rotina mesmo que em alguns casos não seja possível sua efetivação, deve proporcionar atividades que promovam o desenvolvimento das capacidades do educando. Para isso é necessário antes fazer um levantamento de dados sobre o paciente, como: sua idade, sua condição cognitiva e motora, para que possa ser trabalhado a possibilidade de brincar, manipular objetos, e o convívio social.

Segundo Fonseca (2003), o professor é antes de tudo um mediador das interações da criança com o meio hospitalar. Em alguns casos faz intermédio entre os médicos e a família e no que se refere a aprendizagem, entre o paciente e os conteúdos escolares.

\section{CONSIDERAÇÕES FINAIS}

Pode-se compreender que a Pedagogia Hospitalar é um ramo da pedagogia que possui fundamental relevância para os educandos que se encontram em situações hospitalares. Em que vai ser trabalhado além de questões voltados à aprendizagem, como também necessita considerar a condição que ele se encontra, ou seja, é importante perceber o aluno de maneira ampla, utilizando da interação e de momentos prazerosos para o melhor desenvolvimento dentro dos hospitais.

Apesar do contexto no qual essas crianças e adolescentes estão inseridas, as dificuldades, as situações de tratamento em alguns casos é dolorosa, ainda assim é possível oferecer a esses pacientes uma forma de 
escolarização, respeitando os limites de cada uma, para que assim o tratamento seja menos doloroso possível, onde por intermédio dos professores essas crianças e adolescentes ampliem seu desenvolvimento e a pedagogia hospitalar continue promovendo a aprendizagem nesses ambientes.

Neste aspecto, o propósito da Pedagogia Hospitalar está em propor um local que o conhecimento seja alcançado, de maneira que atenda a criança/adolescente, buscando integrar o doente na situação que se encontra.

Assim, é pertinente considerar os diretos que respaldam a criança hospitalizada, para então direcionar e auxiliar frente aos desafios encontrados, bem como, agregar meios que favoreçam um melhor desenvolvimento desse sujeito. Sendo assim, é necessário ter um “olhar" humanizado frente à tais direitos, auxiliando em sua aplicação

Por conseguinte, o profissional que atua na Pedagogia hospitalar pode propor maneiras diferentes de mediar o saber entre os educandos. Dessa maneira será possível aprimorar as capacidades de cada ser, por meio do planejamento, bem como, da análise dos alunos que irá atender, considerando seu contexto e permitindo que a ludicidade tenha espaço neste ambiente hospitalar. O professor atua como mediador entre a criança com o meio em que está inserido, procurando auxiliá-la de forma que ajude a desenvolver suas potencialidades.

\section{REFERÊNCIAS}

LEVY, M. L. Os Direitos da Criança Hospitalizada. Serviço de Pediatria - Hospital de Santa Maria Faculdade de Medicina de Lisboa - Universidade de Lisboa, 1996.

BRASIL. Ministério da Educação. Classe hospitalar e atendimento pedagógico domiciliar: estratégias e orientações. Secretaria de Educação Especial. Brasília: MEC/ SEESP; 2002.

. Direitos da criança e do adolescente hospitalizados. Resolução n. ${ }^{\circ}$ 41, de 13/10/1995. Brasília: Imprensa Oficial, 1995.

. Conselho Nacional dos Direitos da Criança e do Adolescente. Resolução n 41 de Outubro de 1995 (DOU 17/19/95). 
Carta da criança hospitalizada / Instituto de Apoio à Criança. Humanização dos serviços de atendimento à criança. Lisboa: IAC, 1998. 22 p. ISBN 972-8003-14-5.

ESTEVES, C. R. Pedagogia Hospitalar: Um breve histórico, 2008. Disponível em: http://pedagogiaaopedaletra.s3.amazonaws.com/wp-content/ uploads/2013/06/HIST\%C3\%93RICO-DA-PEDAGOGIA-HOSPITALAR.pdf>. Acesso em: 27 mai 2016.

FONSECA, E. S. A situação brasileira do atendimento pedagógico-educacional hospitalar. Disponível em: <http://www.scielo.br/scielo. php? script $=$ sci_arttext\&pid=S1517-97021999000100009>. Acesso em: 25 mai 2016.

Classe hospitalar: Ação sistemática na atenção às necessidades pedagógico-educacionais de crianças e adolescentes hospitalizados, São Paulo, Universidade de São Paulo, 1997. Disponível em: <http://www. gestaoescolar.diaadia.pr.gov.br/arquivos/File/pdf/classehospitalar_ene-ida.pf>. Acesso em: 10 jun 2016.

Atendimento escolar no ambiente hospitalar. São Paulo: Memnon, 2003.

FONTES, R. S. A reinvenção da escola a partir de uma experiência instituinte em hospital. São Paulo:2004. Disponível em: <http://www. scielo.br/pdf/ep/v30n2/v30n2a05.pdf>. Acesso em: 10 jun 2016.

FREITAS, S.N. e ORTIZ, L.C.M. Classe Hospitalar: Caminhos Pedagógicos entre saúde e educação. Editora UFSM, 2005;

FROEBEL, F. W. A. A educação do homem. Tradução de Maria Helena Câmara Bastos. Passo Fundo: UFP, 2001.

LIMA, C. C. F. e PALEOLOGO, S. O. A. Pedagogia Hospitalar: A importância do apoio pedagógico dentro dos hospitais para jovens e crianças. Disponível em: <http://www.faceq.edu.br/e-faceq/downloads/numero01/pedagogia $\% 20$ hospitalar $\% 20$ cristina $\% 20$ cavallari.pdf $>$. Acesso em: 21 jun 2016 
MATOS, E. L. M. e MUGiATTI, M. M. T. F. Pedagogia Hospitalar: A Humanização Integrando Educação e Saúde. 4. ed. Rio de Janeiro. Ed. Vozes. 2009.

Pedagogia em Ação, Revista Eletrônica do Curso de Pedagogia, Pontifícia Universidade Católica de Minas Gerais, v. 1, n. 1, $1^{\text {o }}$ sem. 2009.

PIMENTA, S. G. Pedagogia e Pedagogos: caminhos e perspectivas. São Paulo: Cortez, 2001.

Recebido em: 20/02/2018 Aceito em: 27/03/2018 